\title{
The metallicity of gamma-ray burst environments from high-energy observations
}

\author{
D. Watson ${ }^{1}$ and P. Laursen ${ }^{2}$ \\ 1 Dark Cosmology Centre, Niels Bohr Institute, University of Copenhagen, Juliane Maries Vej 30, 2100 Copenhagen Ø, Denmark \\ e-mail: darach@dark-cosmology.dk \\ 2 Oskar Klein Centre, Dept. of Astronomy, Stockholm University, AlbaNova, 10691 Stockholm, Sweden \\ e-mail: plaur@astro.su.se
}

Received 2 November 2010 / Accepted 4 Janvier 2011

\begin{abstract}
Gamma-ray bursts (GRBs) and their early afterglows ionise their circumburst material. Only high-energy spectroscopy therefore, allows examination of matter close to the burst itself. Soft X-ray absorption allows an estimate to be made of the total column density in metals. The detection of the X-ray afterglow can also be used to place a limit on the total gas column along the line of sight based on the Compton scattering opacity. Such a limit would enable, for the first time, the determination of lower limits on the metallicity in the circumburst environments of GRBs. In this paper, we determine the limits that can be placed on the total gas column density in the vicinities of GRBs based on Compton scattering. We simulate the effects of Compton scattering on a collimated beam of high-energy photons passing through a shell of high column-density material to determine the expected lightcurves, luminosities, and spectra. We compare these predictions to observations, and determine what limits can be placed on the total gas column density. The smearing out of pulses in the lightcurve from Compton scattering is not likely to be observable, and its absence does not place strong constraints on the Compton depth. However, the distribution of observed luminosities of bursts allows us to place statistical, model-dependent limits that are typically $\lesssim 10^{25} \mathrm{~cm}^{-2}$ for less luminous bursts, and as low as $\sim 10^{24} \mathrm{~cm}^{-2}$ for the most luminous. By using the shape of the high-energy broadband spectrum, however, limits as low as $\sim 5 \times 10^{24} \mathrm{~cm}^{-2}$ can be placed on individual bursts, implying metallicity lower limits from $X$ - and gamma-rays alone from 0 up to $0.01 Z / Z_{\odot}$. At extremely high redshifts, this limit would be at least $0.02 Z / Z_{\odot}$, enough to distinguish population III from non-primordial GRBs.
\end{abstract}

Key words. gamma-ray burst: general - gamma-ray burst: individual: GRB 050904 - early Universe - galaxies: ISM dark ages, reionisation, first stars - stars: Population III

\section{Introduction}

A major issue in modern cosmology is our understanding of the metal-enrichment history of the universe: how, when, and where the non-primordial elements were synthesised. Most avenues open to us to investigate this question at high redshifts rely on bright sources such as active galactic nuclei and gammaray bursts (GRBs) as back-lighting for absorption spectroscopy of distant galaxies. The afterglows of GRBs are bright enough to examine the contents of star-forming galaxies by absorption spectroscopy. Most detailed information so far has come from optical and ultraviolet (UV) observations. Due to the strong ionising effect of the burst and early afterglow on the matter surrounding a GRB, however, only high-energy spectroscopy can probe the matter close to the burst. X-ray spectra allow examination of this matter, which we now know lies within only a few parsecs (Watson et al. 2007) and is ionised to a high degree (Prochaska et al. 2008; Schady et al. 2011) and is therefore invisible at optical and UV wavelengths.

Photoelectric absorption by inner shell electrons absorb $\mathrm{X}$-rays and this allows an estimate to be made of the total column density in metals. The total gas column is unavailable, however, since the hydrogen, which is ionised, is transparent to the X-rays even at moderate column densities. However, at extreme column densities, ionised hydrogen is no longer transparent to high-energy photons since the X-rays scatter off the free electrons with a cross section approximated by the Thomson cross section at low energies, $\sigma_{\mathrm{T}} \sim 6.65 \times 10^{-25} \mathrm{~cm}^{2}$. This fact has been used to derive limits on the column density of electrons and hence ionised hydrogen in the immediate surroundings of the GRB 050904 (Campana et al. 2007), and to exclude Comptonisation as the origin of very hard gamma-ray emission in some GRBs (Ghirlanda et al. 2003). Indeed, Compton attenuation was proposed and tested as an explanation of the high-energy emission from cosmological GRBs (Brainerd et al. 1998). It has also recently been suggested by Campana et al. (2011) that the detection of the X-ray and gamma-ray emission from GRBs could be used to place a limit on the total gas column along the line of sight based on the fact that at very high column densities Compton scattering will essentially eliminate all of the X-and gamma-ray emission and that this could be useful for future GRB missions currently in the planning stages (e.g. Campana et al. 2011). Such a limit would allow us to estimate lower limits to the metallicity in the immediate environments of GRBs by providing an upper limit to the total gas column density. At high redshifts, not only could this allow us to determine metallicities without the problems associated with UV spectra, but it would also allow us to determine the metal abundances right in the hearts of star-forming regions.

Future X-ray missions with large effective area, highresolution spectrographs would allow redshifts to be determined directly from X-rays without reference to optical or UV light, enabling far more complete and unbiased estimates of GRB 
environments to be obtained. However, without this technique, they could never determine the metallicities of the regions they probe. In placing this Compton-thick limit, however, Campana et al. (2011) assume for simplicity that this limiting column density of electrons and hence of ionised hydrogen, is approximately one optical depth $\tau=1 / \sigma_{\mathrm{T}}$. But given that the observed column densities of GRBs from metals span a range from $<10^{21} \mathrm{~cm}^{-2}$ up to $\sim 10^{23} \mathrm{~cm}^{-2}$ (Evans et al. 2009; Campana et al. 2010, in units of equivalent hydrogen column density for solar metallicity), the value of the limit that can be placed on the total hydrogen column density makes a substantial difference to how interesting the metallicity lower limit obtained will be. In this paper we quantify the limits that can placed on the total column density of electrons, and hence metallicities of the circumburst medium from the Compton-transparency of GRBs and their afterglows.

\section{Simulations}

\subsection{Burst modeling}

The radiative transfer (RT) calculations are conducted using a modified version of the Monte Carlo RT code MoCaLaTA, originally designed for simulating the scattering of Ly $\alpha$ photons in the interstellar and intergalactic media (Laursen et al. 2009). Although the code is capable of assuming an arbitrary distribution of gas density, temperature, velocity field, etc., taken e.g. from a hydrodynamic simulation, for the purpose of the present calculations the burst is modeled as a central source emitting a power-law spectrum of photons with energies $E$ between $0.1 \mathrm{keV}$ and $1 \mathrm{MeV}$ and a photon spectral index of $\Gamma=2$. That is, the emitted spectrum is given by

$$
F_{\mathrm{em}}(E)=A E^{-\Gamma},
$$

where $A$ is a constant. The source is surrounded by a thick, spherical shell of electron column density $N_{\mathrm{e}}$, with a temperature $T$ and velocity field $\boldsymbol{v}_{\text {bulk. }}$. Photons are emitted in an instantaneous burst in two narrow cones of opening angle $\vartheta=5^{\circ}$, taken to lie along the $z$ axis.

\subsection{Radiative transfer}

While at low energies a photon interacting with an electron is scattered in a more or less random direction, at high energies the phase function is characterised by a significant probability of forward scattering. Moreover, the cross section $\sigma_{\mathrm{C}}$ decreases with increasing photon energy. These effects are given by the differential cross section (Klein \& Nishina 1929)

$$
\frac{\mathrm{d} \sigma_{\mathrm{C}}}{\mathrm{d} \Omega}=\frac{1}{2} r_{\mathrm{e}}^{2} P^{2}(E, \theta)\left(P(E, \theta)+\frac{1}{P(E, \theta)}-1+\cos ^{2} \theta,\right)
$$

where $r_{\mathrm{e}}$ is the classical electron radius, and

$$
P(E, \theta)=\frac{1}{1-\alpha(1-\cos \theta)}
$$

is the energy ratio of the incident and the scattered photon, with $\alpha \equiv E / m_{\mathrm{e}} c^{2}$ being the photon energy in terms of the electron rest energy.

The photons are followed as they scatter stochastically through the medium (or escape freely). Equations (2) and (3) are expressed in the reference frame of the electron. Thus, at each scattering the energy of a photon is first Lorentz transformed to

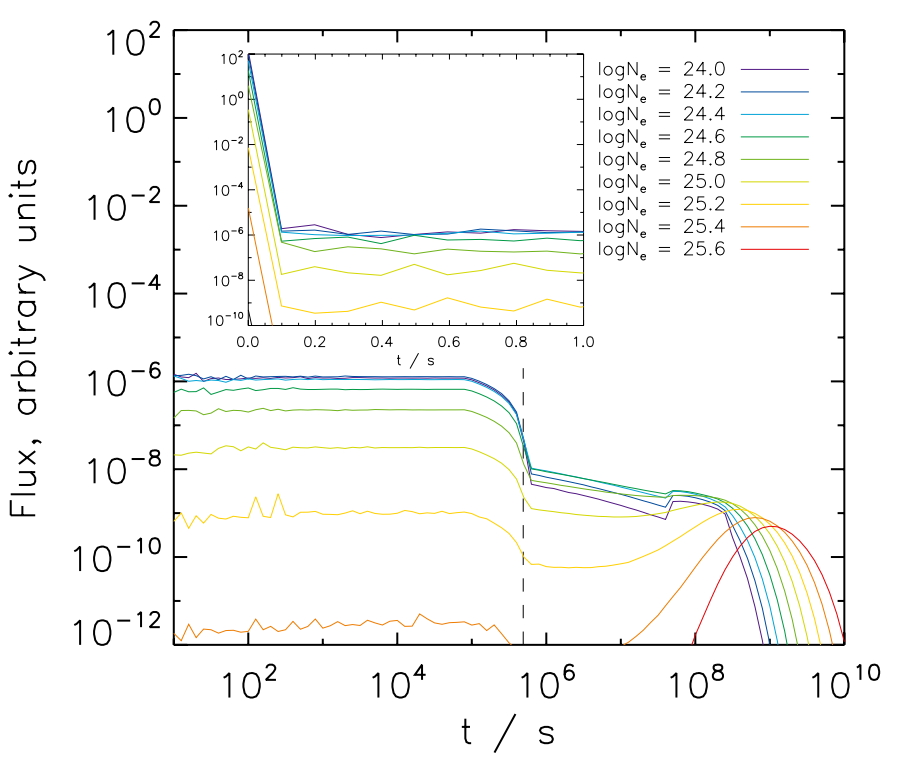

Fig. 1. Lightcurves for a range of electron column densities $N_{\mathrm{e}}$. Only the prompt emission will be detectable with current instrumentation for cosmological GRBs. The inset is a zoom-in of the prompt emission. The vertical dashed line marks the maximum time lag for singly scattered photons, as given by Eq. (4).

the reference frame of the electron, given by the sum of $\boldsymbol{v}_{\text {bulk }}$ and a thermal velocity drawn from a Gaussian distribution. At high energies the change in energy is dominated by the Compton effect described by Eq. (3); however, at lower energies the Doppler shift induced by high velocity electrons may become important.

MoCaLaTA has already been tested extensively against analytical solutions; the additional subroutines implemented in this study were correspondingly tested. Furthermore, a variety of temperatures, velocity and density fields, and opening angles were tested. However, as will be shown in Sect. 3.1, observationally only the prompt emission is of interest, which essentially depends on the column of intervening gas and not its structure.

\section{Results}

\subsection{Lightcurves}

Sampling the photons according to the time it takes to reach the observer, a lightcurve can be obtained. Figure 1 shows such lightcurves for a range of electron column densities. The curves are characterised by three parts of different physical origin: the initial peak - the "prompt" emission - consists of photons that escape the medium directly, without scattering, and hence its height is simply specified by the optical depth along the line of sight to the burst. The following intermediate phase consists of photons that are scattered once, and thus its extension is given by the size of the cloud and the opening angle of the jet; for a spherical cloud of radius $R=1.25 \mathrm{pc}$ and opening angle $\vartheta=5^{\circ}$, this phase lasts

$$
\Delta t=\frac{R}{c}(1-\cos \vartheta) \simeq 5.67 \mathrm{~d} .
$$

Finally, there is an afterglow of photons that scatter several times and thus perform a random walk out of the cloud. Since the number of steps in a random walk scales with $N_{\mathrm{e}}^{2}$, but the step size decreases linearly with $N_{\mathrm{e}}$ for a fixed size of the cloud, the maximum of the afterglow approaches a time proportional to $N_{\mathrm{e}}$. 
D. Watson and P. Laursen: Metallicity of GRB environments using $\gamma$-rays

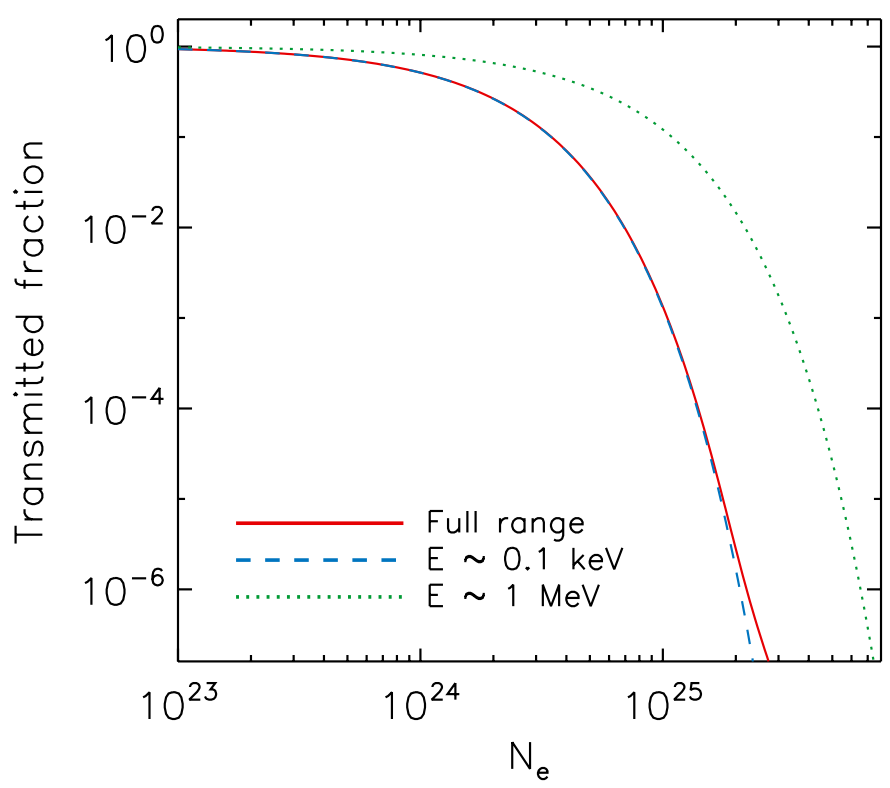

Fig. 2. Fraction of emitted photons that are transmitted directly through a cloud of column density $N_{\mathrm{e}}$ for the full energy range (red solid), lowenergy photons (blue dashed), and high-energy photons (green dotted).

For high values of electron column density, the above considerations become somewhat inaccurate, since at each scattering, a photon - especially a high-energy photon - loses energy, thus resulting in a slowly decreasing electron cross section.

Comparing the magnitude of the observed simulated prompt peak with the energy emitted by the burst, constraints can be put on the Compton thickness of the circumburst material. For the emitted spectrum in Eq. (1), the observed spectrum of the prompt emission will be

$$
F_{\text {obs }}(E)=A E^{-\Gamma} \mathrm{e}^{-N_{\mathrm{e}} \sigma_{\mathrm{C}}(E)} .
$$

The fraction $f$ of the flux observed as prompt emission is thus

$$
f=\frac{\int_{E_{\min }}^{E_{\max }} E^{-\Gamma} \mathrm{e}^{-N_{\mathrm{e}} \sigma_{\mathrm{C}}(E)} \mathrm{d} E}{\int_{E_{\min }}^{E_{\max }} E^{-\Gamma} \mathrm{d} E} .
$$

This fraction is shown as a function of electron column density in Fig. 2.

\subsection{Spectra}

Due to the decreasing Compton cross section with photon energy, an emitted spectrum of constant $\Gamma$ will have its high-energy end transmitted more efficiently than its low-energy end, resulting in a harder slope at high energies (Fig. 3). In practice, this means that the spectral slope will change as a function of energy. Figure 4 shows how the observed spectral slope $\Gamma_{\text {obs }}$ changes with optical depth. Assuming an intrinsically energyindependent spectral slope $\Gamma$, if a broad energy range is observed the change in $\Gamma_{\text {obs }}$ as a function of energy reveals the optical depth of the intervening cloud. For example, for $\Gamma=2$ a column density of $N_{\mathrm{e}} \simeq 10^{25} \mathrm{~cm}^{-2}$ will result in an observed slope $\Gamma_{\text {obs }} \lesssim 1$ at high energies.

\section{Discussion}

To determine limits on the Compton depth of a given burst we have three diagnostics at our disposal: the lightcurve, the total

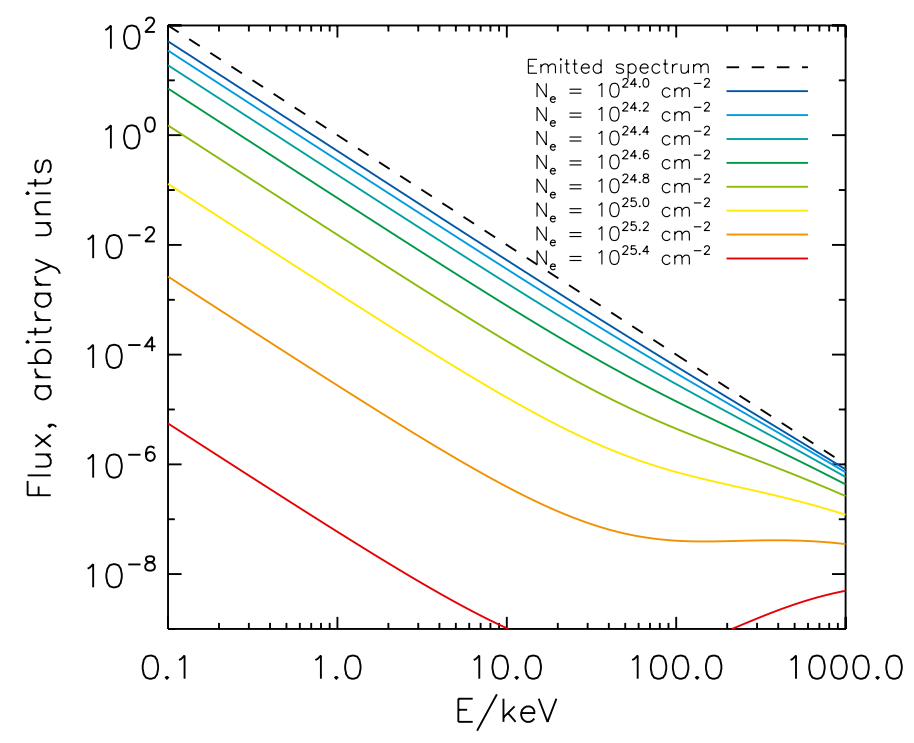

Fig. 3. The model input and output spectra for the simulated GRB afterglow for a range of foreground electron column densities.

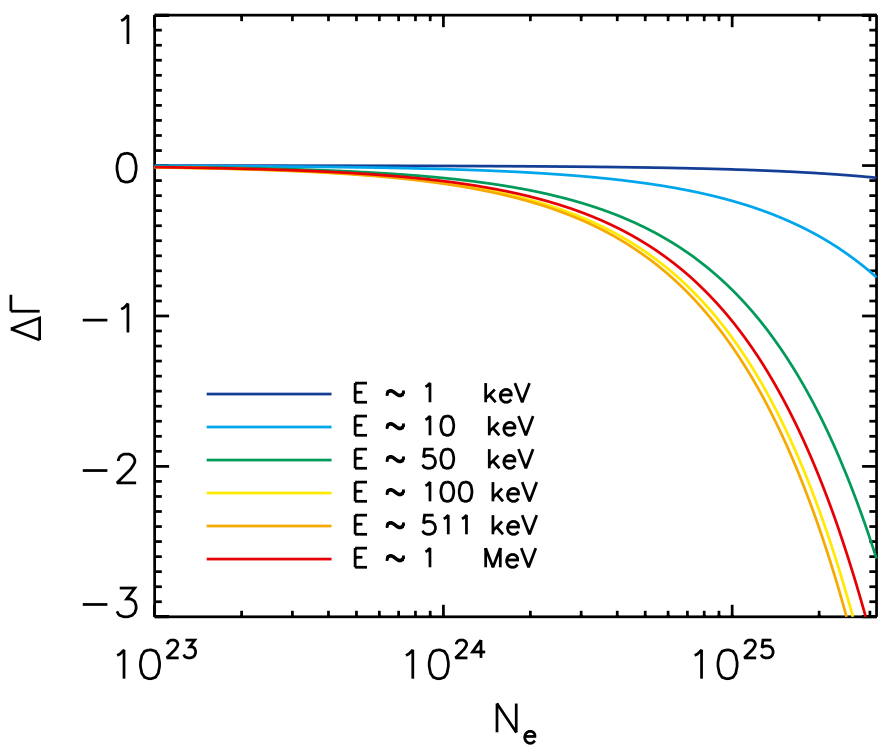

Fig. 4. Change, $\Delta \Gamma$, in the observed spectral slope $\Gamma_{\mathrm{obs}}$ as a function of column density $N_{\mathrm{e}}$, at various energies.

luminosity, and the spectrum. We have shown that the flux in the lightcurve (and the fluence over the observed timescales of GRBs), is dominated by the directly transmitted component even up to high column densities $\left(N_{\mathrm{e}} \gtrsim 3 \times 10^{25} \mathrm{~cm}^{-2}\right.$, see Fig. 1). Smearing of the shape of the lightcurve, therefore, will not enable one to discern even moderately high column density bursts.

The limits that can be placed on the electron column density based on the luminosity of a burst are not very precise because we do not know intrinsically what the luminosity of a burst was. We do, however, have a distribution of equivalent isotropic luminosities of GRBs, and we know that bursts with luminosities above $\sim 2 \times 10^{54} \mathrm{erg}$ are rarely detected (Ghirlanda et al. 2008). If we assume that there is no bias relating luminous bursts with high column density sightlines, it suggests that any burst is likely to be intrinsically less luminous than this value. We can therefore use the luminosity of a burst to determine the diminution in flux 
possibly attributable to Compton scattering. In Fig. 2 we show the fraction of emitted photons in the prompt emission as a function of electron column density. In the general case at high redshift, we are unlikely to detect any burst more than two orders of magnitude fainter than $10^{54} \mathrm{erg}$ with Swift-BAT, and so the electron, and hence hydrogen, column density for high-redshift Swift bursts must always be $\lesssim 10^{25} \mathrm{~cm}^{-2}$ (see Fig. 2). In the specific case of the $z=6.3$ GRB 050904 , this would imply a maximum column density of only a few times $10^{24} \mathrm{~cm}^{-2}$ because of the high apparent luminosity of this burst. However, individual cases are always vulnerable to the argument that they may have an exceptional intrinsic luminosity. Even in the general case, it is possible that $z>6$ GRBs may be inherently more luminous than lower redshift GRBs, weakening this argument further. It might be possible to predict the apparent luminosity of a burst based on a number of correlations of burst properties with luminosity, the most well-regarded of which is the peak energy-isotropic luminosity correlation (the "Amati relation", see Ghirlanda et al. 2008, and references therein). However, at the moment none of these correlations is very tight and their general validity is controversial. We therefore turn to the spectra to examine whether they can provide more robust constraints.

The spectra show a clear deviation from a power-law at column densities above $N_{\mathrm{e}} \sim 10^{25} \mathrm{~cm}^{-2}$, if we have simultaneous broadband coverage from a few $\mathrm{keV}$ to a few hundred $\mathrm{keV}$ (see e.g. Brainerd et al. 1998, for previous work on fitting GRB broadband spectra with Compton attenuation, with a different perspective). Currently, we do not typically obtain simultaneous soft and hard X-ray detections of a GRB, though there are a few exceptions where the burst was long and the Swift response time short. A good example is, in fact, GRB 050904 where the spectrum of the late prompt phase is detected in the range $2-700 \mathrm{keV}$ in the rest frame (Watson et al. 2006; Cusumano et al. 2007).

In general, at $10^{25} \mathrm{~cm}^{-2}$, the spectral slope has changed by 1 in the $0.1-1 \mathrm{MeV}$ range (Fig. 4). Such a strong change in the slope of a burst would be readily discernible in late prompt data with simultaneous X-and gamma-ray spectra, and it seems likely that this spectral characteristic represents the strongest constraint on the column densities. It should be cautioned that in most GRBs strong intrinsic spectral evolution of the burst occurs, causing the low-energy data to change spectral slope substantially. In the case of GRB 050904, the slope changes in the first few minutes from $\Gamma \sim 1.2$ to $\Gamma \sim 1.9$. Such early spectral evolution is common in GRBs. It is therefore essential that in looking for this effect the high- and lowenergy data be simultaneous. Using GRB 050904, we note that the BAT and XRT contemporaneous spectra have compatible power-law slopes, with uncertainties of $\sim 0.1$ on their spectral indices (Cusumano et al. 2007). Disregarding the relatively small flux and cross-calibration uncertainties, this similarity of the observed spectral slopes between the XRT and BAT yields a $3 \sigma$ limit on the column density of $N_{\mathrm{e}} \sim N_{\mathrm{H}} \sim 5 \times 10^{24} \mathrm{~cm}^{-2}$. This is a tight constraint, but still three times as high as the $\tau=1$ limit imposed by (Campana et al. 2007) and yields therefore a metallicity limit of $>1 \%$ of the solar value (90\% confidence, following their analysis of the X-ray absorption which assumed solar ratio abundances for the metals, except Fe which was set to zero). It is also worth noting that the detection of the high column density in GRB 050904 is disputed (Butler \& Kocevski 2007), and there is the risk of confusing intrinsic spectral curvature for photoelectric absorption unless high signal-to-noise ratio data at multiple epochs is available or additional uncertainty is allowed into the absorption fit by using an intrinsically curved spectral model.

\subsection{Population III progenitors}

At very high redshifts, GRBs may be formed from primordial stars - population III stars. If a GRB occurs at very high redshift, a discriminator between a population III GRB and a nonprimordial GRB would be useful. At $z=9-13$, for example, the lowest feasible detection of photoelectric absorption is $\sim 10^{23} \mathrm{~cm}^{-2}$ in units of equivalent hydrogen column density at solar metallicity. Assuming a similar limit on the total electron column density as found for GRB 050904, of $\sim 5 \times 10^{24} \mathrm{~cm}^{-2}$, where soft X-ray absorption is detected, this would imply a metallicity limit at least $\gtrsim 0.02 Z / Z_{\odot}$ in the $\sim 1$ pc environment of the GRB. This should be sufficient to exclude a population III star as the GRB progenitor.

\section{Conclusions}

We have examined the prospect of using only the high-energy emission of GRBs to place a lower limit on metallicities around them; the soft X-ray photoelectric absorption providing a measure of the total metal column density and the Compton thickness limit a maximum column density of electrons and hence hydrogen. We find that smearing of the lightcurves does not provide strong constraints on the column densities, while apparent luminosity and deviations from a power-law spectral shape provide stronger constraints. The spectral constraints are more reliable, but require the assumption of a single power-law spectral shape and simultaneous gamma- and X-ray coverage. The apparent luminosity constraints are more readily applicable, but are less certain, and rely heavily on an assumption that we know the distribution of apparent luminosities of GRBs, which is especially uncertain at high redshift. We find for the individual case of GRB 050904, the best limit that can be obtained from spectra corresponds to $\sim 5 \times 10^{24} \mathrm{~cm}^{-2}$, three times what was previously assumed. While the results are not very constraining for most GRBs, with bright, long-lasting bursts, with detections of very high metal column densities, the method could be used to exclude a population III progenitor for a high redshift burst.

Acknowledgements. The Dark Cosmology Centre is funded by the DNRF. P.L. acknowledges funding from the Villum Foundation. The simulations were performed on the facilities provided by the Danish Center for Scientific Computing. We would like to thank Jens Hjorth and Anja C. Andersen for a critical reading of the manuscript.

\section{References}

Brainerd, J. J., Preece, R. D., Briggs, M. S., Pendleton, G. N., \& Paciesas, W. S. 1998, ApJ, 501, 325

Butler, N. R., \& Kocevski, D. 2007, ApJ, 663, 407

Campana, S., Lazzati, D., Ripamonti, E., et al. 2007, ApJ, 654, L17

Campana, S., Thöne, C. C., de Ugarte Postigo, A., et al. 2010, MNRAS, 402, 2429

Campana, S., Salvaterra, R., Tagliaferri, G., Kouveliotou, C., \& Grindlay, J. 2011, MNRAS, 410, 1611

Cusumano, G., Mangano, V., Chincarini, G., et al. 2007, A\&A, 462, 73

Evans, P. A., Beardmore, A. P., Page, K. L., et al. 2009, MNRAS, 397, 1177

Ghirlanda, G., Celotti, A., \& Ghisellini, G. 2003, A\&A, 406, 879

Ghirlanda, G., Nava, L., Ghisellini, G., Firmani, C., \& Cabrera, J. I. 2008, MNRAS, 387, 319

Klein, O., \& Nishina, T. 1929, Zeitschrift fur Physik, 52, 853

Laursen, P., Razoumov, A. O., \& Sommer-Larsen, J. 2009, ApJ, 696, 853

Prochaska, J. X., Dessauges-Zavadsky, M., Ramirez-Ruiz, E., \& Chen, H. 2008, ApJ, 685, 344

Schady, P., Savaglio, S., Kruehler, T., Greiner, J., \& Rau, A. 2011, A\&A, 525, A113

Watson, D., Reeves, J. N., Hjorth, J., et al. 2006, ApJ, 637, L69

Watson, D., Hjorth, J., Fynbo, J. P. U., et al. 2007, ApJ, 660, L101 